\title{
Parton densities from DIS and hadron colliders to LHC: WG summary
}

\author{
Sergey Alekhin ${ }^{1 *}$, Dimitri Colferai ${ }^{2}$, Joey Huston $^{3}$ and Ringailè Plačakytè $\dot{e}^{4 \dagger}$ \\ 1 - Deutsches Elektronen-Synchrotron DESY, \\ Platanenallee 6, D-15738 Zeuthen, Germany \& \\ Institute for High Energy Physics IHEP, \\ Pobeda 1, 142281 Protvino, Russia. \\ E-mail: sergey.alekhin@ihep.ru \\ 2 - University of Florence, \\ Department of Physics, \\ Via G. Sansone 1, I-50019 Sesto Fiorentino (Firenze), Italy. \\ E-mail: colferai@fi.infn.it \\ 3 - Michigan State University, \\ Department of Physics and Astronomy, \\ 3218 Biomedical Physical Science Building, \\ East Lansing, MI 48824, USA. \\ E-mail: huston@pa.msu.edu \\ 4 - Deutsches Elektronen Synchrotron DESY, \\ Notkestrasse 85, D-22607 Hamburg, Germany. \\ E-mail: ringaile@mail.desy.de
}

Recent experimental and theoretical results presented in the working group "Parton densities from DIS and hadron colliders to LHC" at the DIS2010 workshop are summarized in this contribution.

XVIII International Workshop on Deep-Inelastic Scattering and Related Subjects April 19 -23, 2010

Convitto della Calza, Firenze, Italy

\footnotetext{
*Speaker.
}

† Speaker. 
Parton distribution functions (PDFs) are determined from global analyses of a wide range of data resulting from a variety of hard-scattering processes; these processes include deep inelastic scattering (DIS) of leptons off of a nucleon, lepton pair production in hadron-hadron collisions (the Drell-Yan process), and jet production in proton-antiproton and lepton-proton collisions (see Table 1). The data used in these global analyses are continually updated, taking advantage both of improvements to the data sets and of progress in the theory. At this workshop, new experimental results from HERA, Fermilab, and JLAB experiments were presented and analyzed in the context of PDF determination [1].

\begin{tabular}{lllll}
\hline Name & Data used & QCD order & Scheme & Reference \\
\hline ABKM & DIS+DY & NLO/NNLO & FFN & {$[2]$} \\
CTEQ & DIS+DY+jets & NLO & GMVFN & {$[3]$} \\
HERAPDF & DIS & NLO & GMVFN & {$[4]$} \\
JR & DIS+DY & NLO/NNLO & FFN & {$[5]$} \\
MSTW & DIS+DY+jets & NLO/NNLO & GMVFN & {$[6]$} \\
NNPDF & DIS+DY+jets & NLO & ZMVFN & {$[7]$} \\
\hline
\end{tabular}

Table 1: Recently published nucleon PDF sets, with a brief description of the data used in the fit, the theoretical accuracy and the factorization scheme employed to model the heavy-quark DIS contribution. The table is taken from Ref. [8].

The large sample of data accumulated in the fifteen years of successful HERA operation provides a comprehensive view of the proton, particularly at small values of the Bjorken variable $x$. The latest HERA results presented in this DIS2010 workshop include (1) the combination of DIS cross sections measured at various proton-beam energies, (2) the extraction of the longitudinal structure function $F_{L}$, and (3) updated neutral current (NC) and charged current (CC) measurements. Recent HERA data measurements have resulted in improved determination of both PDFs and of the weak coupling constants.

A new combined data set on inclusive $e^{+} p$ DIS cross sections measured at different protonbeam energies $\left(E_{p}=920 \mathrm{GeV}, E_{p}=460 \mathrm{GeV}\right.$ and $\left.E_{p}=575 \mathrm{GeV}\right)$ was recently produced by the $\mathrm{H} 1$ and ZEUS Collaborations [9]. The variation of the centre-of-mass energy $\sqrt{s}$ allows for the discrimination of the structure functions $F_{L}$ and $F_{2}$. Since DIS cross sections are proportional to $F_{2}-y^{2} F_{L} /\left(1+(1-y)^{2}\right), F_{L}$ and $F_{2}$ can be extracted from measurements at two or more different values of inelasticity $y=Q^{2} / x s$. The combination of the H1 and ZEUS data includes the crosscalibration of two experiments, and results in an improved precision for the data. The proton structure function $F_{L}$ is extracted from the combined cross sections in the range of $2.5 \leq Q^{2} \leq 800$ $\mathrm{GeV}^{2}$ (see Figure 1).

In addition to the combined HERA data of Ref. [9], a new extended NC measurement of the $e^{+} p$ inclusive cross sections at different proton-beam energies has been obtained by the ZEUS Collaboration [9] For this analysis, satellite vertex events were used to access $Q^{2}$ values down to $5 \mathrm{GeV}^{2}$ for the reduced proton beam energies. This measurement should further improve constraints on $F_{L}$ provided by HERA. 

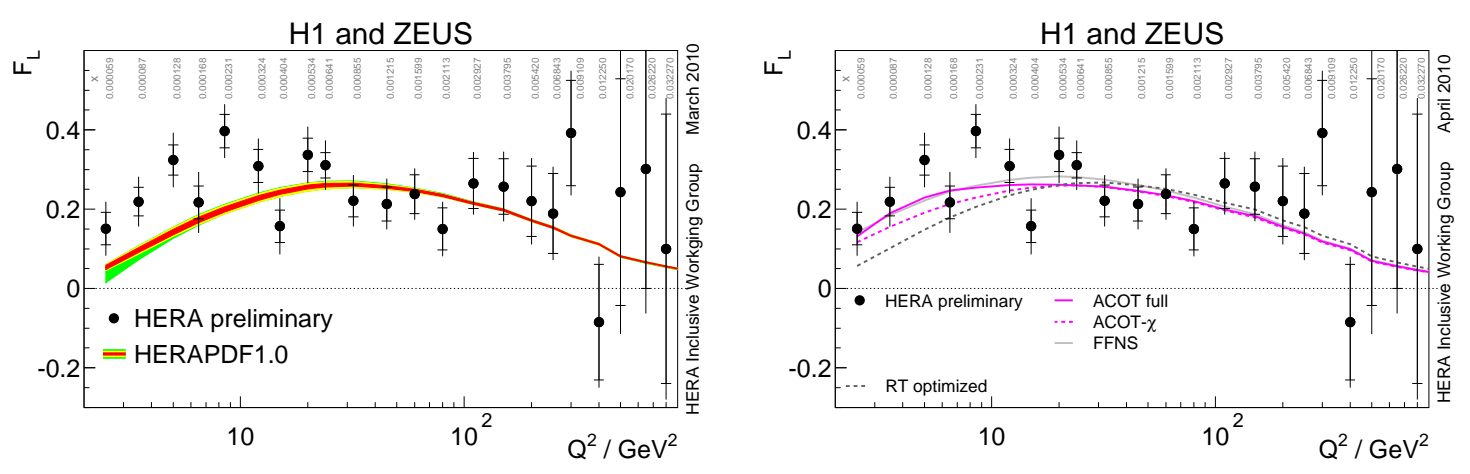

Figure 1: The combined H1 and ZEUS measurement of the structure function $F_{L}$, averaged in $\mathrm{x}$ at a given value of $Q^{2}$, compared to a QCD prediction based on the PDFs of Ref. [4] (left) and different variants of the fits of Ref. [10] (right).

The combined data of Ref. [9] were employed in a QCD fit taking into account QCD corrections up to NNLO [10]. Two variants of the fit with two different cuts on $Q^{2}$ were tried. In this way, the sensitivity of the PDFs to the low $Q^{2}$ portion of the data was determined. The fit is sensitive to the treatment of the heavy quark contribution to the inclusive DIS cross sections. In particular, the predictions based on the optimized Thorne prescription of Ref. [11] undershoot the HERA $F_{L}$ measurement (see Figure 1). The ACOT prescription [12,13] and the (3-flavor) fixed-flavor number (FFN) scheme are in better agreement with the data.

New NC and CC DIS cross section measurements at high $Q^{2}$ were obtained by the H1 and ZEUS Collaborations using the HERA-II sample obtained with longitudinally polarised leptons. These data provide additional constraints on PDFs through the NC polarisation asymmetries and test the chiral structure of the weak interactions.

The ZEUS Collaboration has measured the CC cross sections based on a sample with an integrated luminosity of $132 \mathrm{pb}^{-1}$ and different polarizations of the positron beam [14]. The H1 Collaboration has determined the CC cross sections using the complete HERA-II data sample with polarized $e^{+}$and $e^{-}$beams [15]. In order to achieve better precision in the CC data, the HERA-II CC polarised measurements were combined with the unpolarised HERA-I data. The combined sample corresponds to the total luminosity of $280.8 p b^{-1}$ and $165.5 p b^{-1}$ for $e^{+} p$ and $e^{-} p$ scattering, respectively.

The NC double-differential cross sections for $e^{ \pm} p$ scattering with longitudinally polarised lepton beams were measured by the H1 Collaboration [16]. The charge dependent polarisation asymmetry in the $\mathrm{NC}$ cross sections is sensitive to the quark vector and axial couplings to the $\mathrm{Z}$ boson. Similar to the case of $\mathrm{CC}$ interactions, the $\mathrm{NC}$ cross sections were combined with the earlier H1 measurements. The structure functions $x \tilde{F}_{3}$ and $x F_{3}^{\gamma Z}$ were extracted from the combined unpolarised cross section measurements.

A new method to measure the NC cross sections up to values of $x$ close to 1 was employed by the ZEUS Collaboration [17]. In this region, PDFs are poorly known and the data of Ref. [17] provide valuable additional constraints for the global PDF fits. The NC cross sections were extracted at $Q^{2} \geq 575 \mathrm{GeV}^{2}$ using the $e^{-} p$ collision data sample with an integrated luminosity of $187 \mathrm{pb}^{-1}$. This is a factor of ten larger than the one used for the earlier ZEUS measurement [18]. 
The systematic uncertainties are also reduced as compared to Ref. [18] due to improved kinematic reconstruction methods. The data of Ref. [17] were found to be in good agreement with the predictions based on the CTEQ6D PDFs.

A new combined electroweak and QCD analysis was performed by the H1 Collaboration using the full low and high $Q^{2}$ HERA data [19]. Weak vector and axial couplings of light up- and downtype quarks to the $Z$ boson $\left(v_{q}, a_{q}\right)$ were extracted from the combined fit simultaneously with the PDFs. Due to the additional sensitivity of the polarised NC measurement to the quark vector coupling, the accuracy of the vector coupling $v_{q}$ was improved with respect to earlier results based on the unpolarised HERA data only [20]. The constraint obtained on the up-type quark coupling $v_{u}$ from HERA is better than those obtained from LEP [21] and from the TEVATRON [22] (see Figure 2). Moreover, the HERA data are also sensitive to the sign of weak couplings.
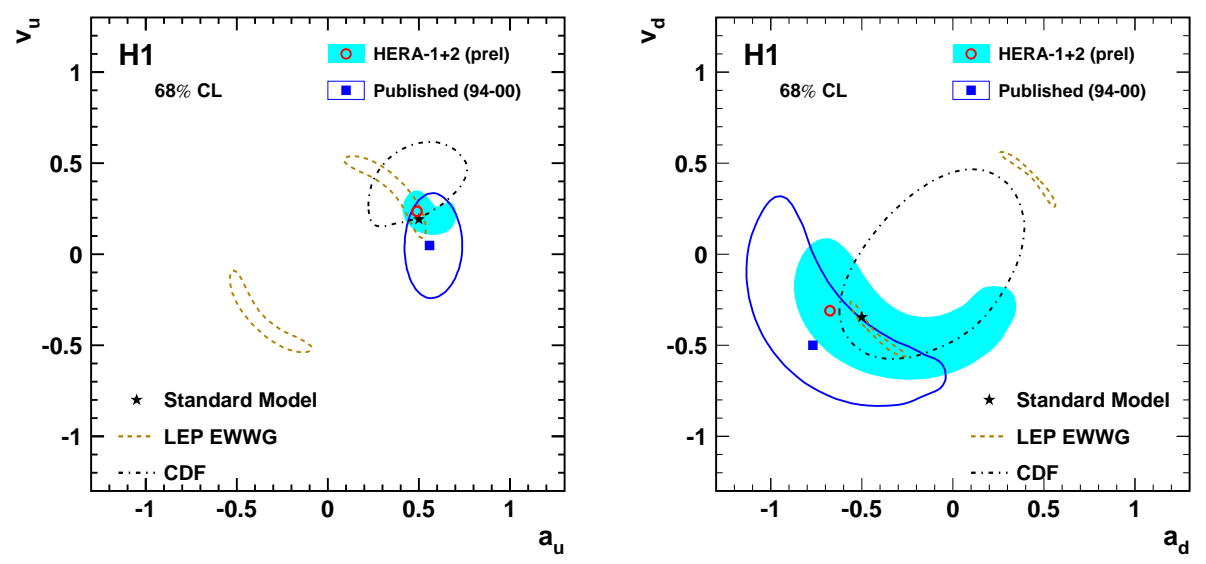

Figure 2: The 68\% confidence level for the determination of the weak couplings of up-(left) and downtype(right) quarks to the $Z$ boson determined in a combined electroweak-QCD analysis using the full HERA H1 data [16]; the results are compared with the corresponding results published previously using the HERA-I data alone [20]; couplings determined by the LEP [21] and TEVATRON [22] are given for the comparison.

The H1 Collaboration presented proton structure function measurements at low and medium $Q^{2}$ determined from the first period of HERA operation. The inclusive double differential cross sections for $e^{+} p$ scattering were obtained at $12 \leq Q^{2} \leq 150 \mathrm{GeV}^{2}$ for an integrated luminosity of $22 \mathrm{pb}^{-1}$ [23]. The data were collected at the beam energies of $\mathrm{E}_{e}=27.6 \mathrm{GeV}$ and $\mathrm{E}_{p}=920$ $\mathrm{GeV}$ and were combined with earlier data obtained with $\mathrm{E}_{p}=820 \mathrm{GeV}$. The typical precision of the combined measurements is $1.3-2 \%$. This is a record level of accuracy for DIS measurements. The NLO QCD fit to the H1 data based on the new measurements provides an improved determination of the gluon and quark densities in the proton, particularly at small x [24]. The analysis of Ref. [24] includes the study of the experimental, model and parameterisation uncertainties in PDFs.

The inclusive NC double differential cross sections of $e^{+} p$ scattering at HERA were obtained by the H1 Collaboration at small $x$ and low $Q^{2}\left(0.2 \leq Q^{2} \leq 12 \mathrm{GeV}^{2}\right)[25,26]$. These data were collected in two dedicated periods with nominal and shifted interaction vertex, where a shifted vertex provided better acceptance at low $Q^{2}$. The two samples overlap at $0.5 \leq Q^{2} \leq 3.5 \mathrm{GeV}^{2}$, and the 
proton structure functions $F_{2}$ and $F_{L}$ were extracted from the combination of these measurements. The data at $Q^{2} \lesssim 2 \mathrm{GeV}^{2}$ correspond to the transition between DIS and photoproduction regimes. With the improved data accuracy, one can discriminate between different theoretical approaches used to model $F_{2}$ and $F_{L}$ in this region [26].

The inclusive $e^{ \pm} p$ NC and CC cross sections measured by the $\mathrm{H} 1$ and ZEUS Collaborations in the first period of HERA operation were combined into a common data set [27]. The combined data cover the range of $6 \cdot 10^{-7}<x<0.65,0.045 \leq Q^{2} \leq 30000 \mathrm{GeV}^{2}$ for the $\mathrm{NC}$ and $0.013<x<0.4$, $300 \leq Q^{2} \leq 30000 \mathrm{GeV}^{2}$ for the CC scattering, respectively. The H1 and ZEUS input data used in the combination were found to be consistent with each other with $\chi^{2} / D O F=636.5 / 656$. The total uncertainty reached with this combination is $1 \%$ for $\mathrm{NC}$ interactions in the most accurately measured region $\left(20<Q^{2}<100 \mathrm{GeV}^{2}\right)$ [4]. A NLO QCD analysis of the combined $e^{ \pm} p$ scattering cross-section data was performed and a new set of parton distribution functions, HERAPDF 1.0, was obtained from the analysis [4]. The experimental uncertainties in the PDFs were reduced with respect to the earlier H1 and ZEUS PDF sets, due to the improved accuracy in the combined data. The theoretical uncertainties obtained by varying the input assumptions of the fit, e.g. the charm quark mass, were also studied.

Several groups $[7,28-30]$ have recently replaced the inclusive $\mathrm{H} 1$ and ZEUS DIS data of Refs. [31] used in their PDF fits with the combined HERA data of Ref. [4]. The new HERA data are in reasonable agreement with the other data sets used in the fits. Moreover, this replacement leads to an improvement in the small- $x$ gluon and sea uncertainties, typically by the order of $20-30 \%$ (see Figure 3). At scales of $O\left(\mathrm{GeV}^{2}\right)$, the accuracy in the HERA data is better than $2 \%$. Due to such precision, NNLO QCD corrections are needed for the most accurate interpretation of these data. The NNLO (3-loop) corrections to the PDF evolution have been

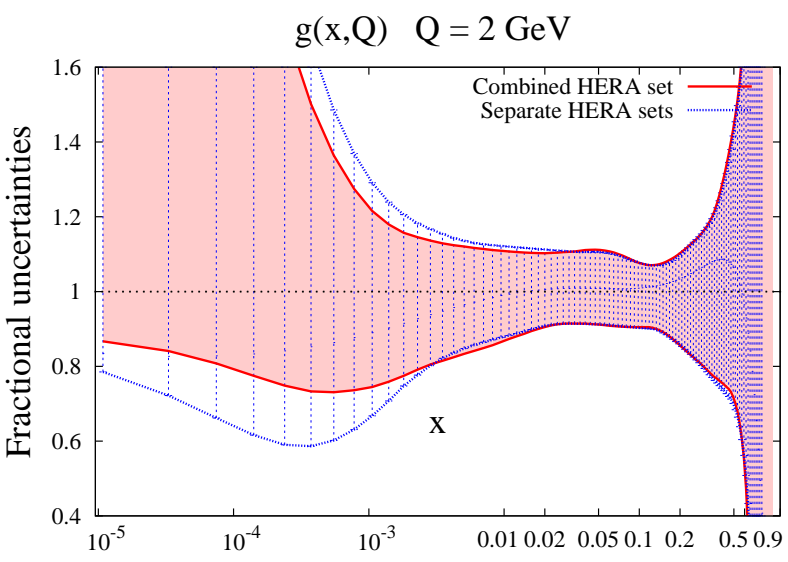

Figure 3: Impact of the combined inclusive HERA data on the fractional gluon uncertainty. Figure taken from Ref. [29]. calculated in Ref. [32]. In addition, the 3-loop corrections to the massless DIS coefficient functions are also known [33]. In the factorization scheme with 3 light flavors in the initial state (the FFN scheme), the corrections to the heavy-quark contribution to DIS are only known up to NLO [34]. This makes an NNLO analysis of the DIS data somewhat inconsistent. The higherorder QCD corrections are partially taken into account through the massless evolution of the heavy quarks, which is employed in the zero-mass variable-flavor-number (ZMVFN) scheme. However, the ZMVFN scheme is applicable only at asymptotically large transfers $Q \gg m_{\mathrm{h}}$, where $m_{\mathrm{h}}$ denotes the heavy quark mass. For realistic kinematics, the exact results are generally overestimated, due to the missed power corrections to the heavy-quark production coefficient functions. This shortcoming is overcome in the general-mass variable-flavor-number (GMVFN) extensions of the 
ZMVFN scheme by a special modeling of the Wilson coefficients at low $Q$. In this way, the GMVFN scheme prescriptions of ACOT, Roberts-Thorne, and Thorne were obtained (cf. review of Ref. [35] and Refs. [11,36] for the recent update of the ACOT and Thorne's prescriptions).

The GMVFN schemes facilitate combination of the DIS and the hadron collider data in the global PDF fits. However, the GMVFN PDFs are sensitive to the specific choice of the prescription. This results to an additional uncertainty in the hard cross section predictions based on these PDFs [37]. The FFN scheme and the different prescriptions of GMVFN schemes provide an equally good description of the combined HERA data on the inclusive and semiinclusive structure functions $F_{2}^{c}$ [38]. Meanwhile, the FFN small- $x$ gluon distribution obtained in the fit of Ref. [38] is substantially higher than gluon distribution determined in the GMVFN variants of the fit (see Figure 4), and is clearly positive at small $x$. In part, this is explained by the definition of the GMVFN PDFs; the remaining discrepancy can be considered as the PDF uncertainty due to the

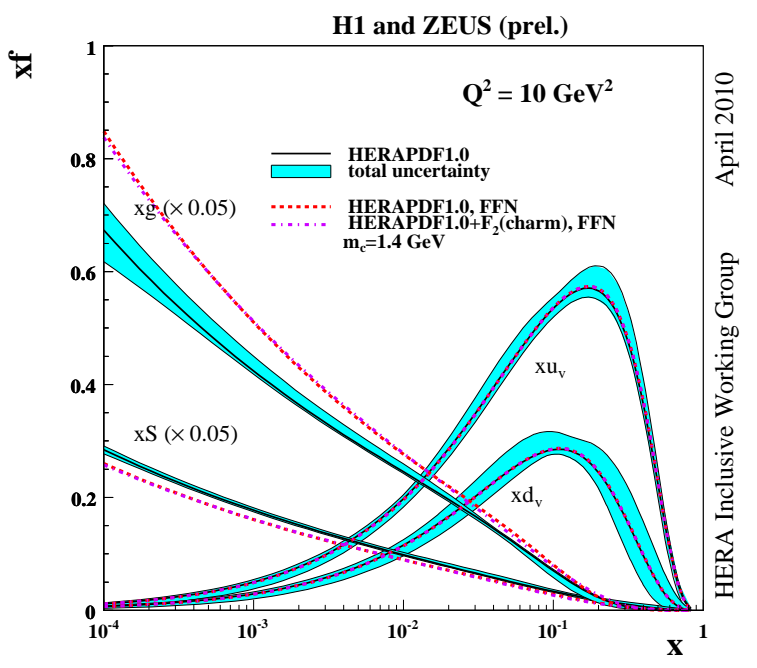

Figure 4: The PDFs obtained in Ref. [4] for the GMVFN scheme of Ref. [11] and in the variant of this fit using the FFN scheme. The figure is taken from Ref. [38]. scheme choice. It is worth noting that the PDFs obtained in the FFN fits correspond to the $\overline{\mathrm{MS}}$ scheme. This is also the case for the GMVFN prescription suggested by Buza-Matiounine-Smithvan Neerven (BMSN) [39] and for the FONLL prescription of Ref. [40], which contains the main BMSN features. This is not generally guaranteed for PDFs obtained within the GMVFN fit formalism [41]. However, the higher-order corrections to heavy-quark DIS production suppress the difference between the FFN and ZMVFN schemes at large $Q$ (see Ref. [42]). For the NLO case, this difference can barely be resolved by the existing data [2] and must be even smaller taking into account the NNLO corrections to the matching conditions for the 4- and 5-flavor distributions [43]. The interpretation of the heavy-quark electro-production and inclusive DIS data also depends on the heavy-quark masses values and their definition. The HERA data alone are not sensitive to the heavy-quark masses since the effect of the mass variation is compensated by changes in the PDFs [10]. The heavy quark masses can be more precisely determined in a global fit including data from other processes. Such a determination was carried out by MSTW and a set of PDFs corresponding to different values of the heavy quark masses is provided in Ref. [44].

Hadron collider data on $W / Z$ and jet production provide more information for PDF determination to one available in the DIS data alone. They provide constraints on PDFs at large factorization scales and at large parton $x$ values and help to disentangle the distributions of different PDF species. Moreover, $W / Z$ production is considered as one of the primary LHC standard candle processes [45], due to the large cross section and small experimental and theoretical uncertainties. The $W / Z$ distributions have been calculated up to NNLO accuracy [46]. The higher-order corrections 
of Refs. [46] are usually employed in the global PDF fits in the form of fixed $K$-factors $[3,6]$ applied to each data point, and updated periodically through the fitting process. In the NNPDF fit [7], the higher order corrections are applied in each fitting iteration with the use of FastKernel tool. A similar technique was employed for the first time in the FastNLO code for the fast calculation of the jet production cross sections [47] and has been developed recently within the APPLGRID project for the NLO QCD analysis of the LHC data on the jet and $W / Z$ production [48].

Recently, precise Run II $W$ lepton asymmetry data have become available [49]. The CTEQ and MSTW collaborations found that the lepton asymmetry data disagree with other data sets used in the global fitting, particularly those from the NMC [50] and BCDMS [51], [52] experiments. The disagreement leads to a non-negligible increase in global $\chi^{2}$ for the global fits, and a poor agreement of the resultant predictions with the lepton asymmetry data. As a result, both CTEQ and MSTW have left the Run II lepton asymmetry data out of their latest global fits. The CTEQ and MSTW predictions, as well as those from ABKM, overshoot the data in general. CTEQ has a variant fit (CT10W) [29] in which the asymmetry data is included in the fit, but given a large

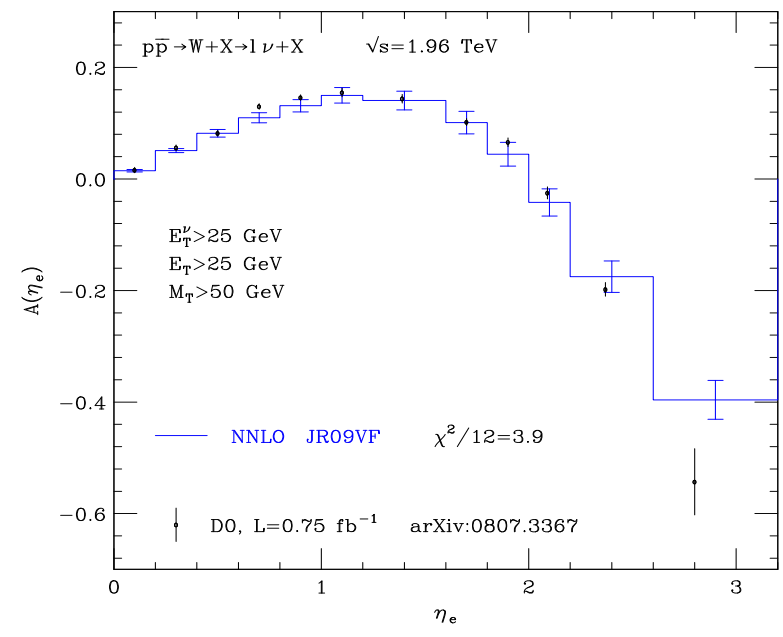

Figure 5: The Run II electron charge asymmetry obtained by the D0 collaboration compared to the NNLO predictions based on the JR PDFs of Ref. [5]. Figure taken from Ref. [53].

weight. This is the only manner in which a reasonable $\chi^{2}$ can be obtained for the asymmetry data sets. The Run II lepton asymmetry analyses have been carried out by both CDF (for electron) and D0 (for both electron and muon). The CDF electron asymmetry data agree with the results from the D0 electron analysis. To make matters more complex, there is some tension between the D0 electron and muon asymmetry data sets. Thus, there is no final conclusion yet as to the impact of the Run II lepton asymmetry data. The best agreement with the data was obtained for the case of the JR PDFs (see Figure 5).

The Tevatron charge asymmetry data are sensitive to the $d$-quark distribution, and to the $d / u$ quark ratio. Therefore, the agreement might be improved due to modification of the correction for nuclear effects in deuterium, which affects the $d$-quark distribution extracted from the deuteron fixed target DIS data that have the conflict with the Run II lepton asymmetry data. A modelindependent form of deuteron correction was attempted in the MSTW fit of Ref. [28]. While this correction somewhat improves the description of the Tevatron charge asymmetry data, the shape of the deuteron correction preferred by this fit cannot be justified by a reasonable nuclear model.

In Ref. [54], nuclear corrections were also fitted to the charged-lepton and neutrino DIS data in the spirit of the nuclear PDF concept [55-57]. In this manner, different PDF shapes were found for neutral-current and charged-current DIS off of an iron target. Therefore the application of the resulting nuclear PDFs to collider predictions is somewhat problematic. Meanwhile, the observation of Ref. [54] is based on the analysis of data from one experiment only; it therefore requires 
independent confirmation. In the model of Ref. [58], the various nuclear effects are considered separately, in contrast to the nuclear PDF approach. This model describes a wide set of nuclear DIS data, including the recent JLAB data for helium-3 [59]. Thus, it can be conjectured that this approach can be also reliably extrapolated to the case of deuteron targets for the benefit of interpretation of the hadron collider lepton asymmetry data.

The production of jets at high transverse momentum in hadron collisions is sensitive to the large- $x$ gluon distribution at both the Tevatron and LHC colliders. The NNLO corrections to the hadronic jet production have not yet been calculated; therefore the jet data can be consistently used only in the NLO version of the PDF fit. For some time, the Run I jet Tevatron data have provided the main constraints on the high $x$ gluon distribution [60,61]. The Run I data, and in particular the Run I data from D0, prefer a higher large- $x$ gluon distribution as compared to the gluon determined from fits to DIS data alone. The Run II inclusive jet data, especially those from D0, are relatively lower at high jet transverse momentum $p_{T}$ and jet rapidity $Y$, and the resultant global fits using this data alone have a lower gluon distribution at high $x$. Thus, there is some tension between the the Run I and the Run II Tevatron jet data. This tension was examined by CTEQ [62]; although some degree of tension does exist, the data sets from Run I and Run II were found to be statistically compatible with each other, with the tensions similar to that between other data sets used in the global fit. Thus, both generations (Run I and Run II) of Tevatron jet data have been kept in recent CTEQ PDF fits [29], although only the Run II data have been used in the MSTW and NNPDF global fits. The resultant high $x$ gluon distribution for CTEQ is thus larger than either MSTW or NNPDF. MSTW has also found the impact of the Run II jet data on the uncertainty in the large- $x$ gluon distribution obtained in the global PDF fits to be relatively minor (see Figure 6), while CTEQ finds the constraints to still be appreciable.

A substantial fraction of jets produced in the forward direction results from the simultaneous scattering of two pairs of partons. The rate for such processes is defined by double-parton distribution functions (dPDFs), i.e. the probability to find two partons with certain momenta inside the nucleon. Due to experimental constraints that are currently insufficient, the dPDFs are usually derived as a product of conventional collinear PDFs. However, the dPDFs obtained in such a way do not enjoy the fermion and momentum sum rules fulfilled for the dPDFs evolution equations. Addressing this shortcoming, a novel set of dPDFs was generated with the MSTW08 PDFs taken as an input [63].

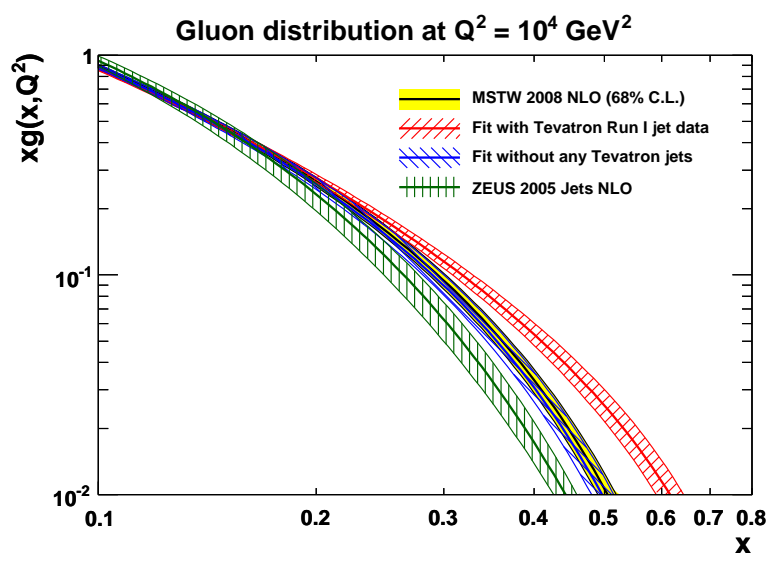

Figure 6: The large- $x$ gluon distribution obtained in the different variants of the NLO MSTW fit. Figure taken from Ref. [6].

For many important processes the fixed-order perturbative QCD calculations demonstrate excellent convergence [64] and in most cases the NNLO approximation is sufficient to meet the data accuracy. However, at small $x$ the perturbative corrections are unstable. In particular, the 3-loop 
terms in the massless DIS coefficient functions are quite large [33]. On the other hand, the small- $x$ resummation of the splitting functions and the DIS Wilson coefficients softens the small- $x$ terms and compensate to some extent the NNLO corrections $[65,66]$. The impact of small- $x$ resummation in DIS was examined with a variant of the NNPDF1.0 PDFs [67] fitted to the high- $Q$ part of the DIS data, and then extrapolated to the low- $Q$ region with the heavy-quark contribution calculated in the ZMVFN scheme [68]. In this way, possible deviations from the standard NLO evolution of the inclusive HERA data were found. The effect observed is explained in Ref. [68] by the impact of small- $x$ resummation or by parton saturation [69]. In line with this observation, the GMVFN variant of the HERAPDF fit of Ref. [10] is sensitive to the low- $Q$ part of the HERA data; however the FFN variant of the fit is much more stable to the cut on $Q$. Resummation effects in DIS thus still need to be systematically explored. This is an important issue for collider phenomenology since resummation contributes to many important collider channels such as heavy-quark and the lepton pair production (cf. Ref. [70] for a recent study of the effects of resummation in direct photon production). Small- $x$ dynamics is often considered within the framework of non-collinear PDF evolution, using $k_{T}$ or angular ordering. The unintegrated parton distributions appear in the parton evolution with the angular ordering depending on both longitudinal and transverse variables; therefore, the calculation of final state transverse momentum distributions is better suited for this formalism. With the appearance of the combined HERA I data, a determination of the unintegrated gluon distribution was updated and found enhanced as compared to the previous determination [71].

PDF shapes cannot be calculated in perturbative QCD. Instead, usually they are parameterized in a model-independent way with loose constraints imposed on the high- $x$ and the low- $x$ PDF exponents, coming from quark counting rules and Regge phenomenology, respectively [2-6]. If the PDFs are evolved starting from a scale $\mu_{\mathrm{F}}^{0}<1 \mathrm{GeV}$, like in the case of JR PDFs, at the hard process scales they enjoy the asymptotic behavior defined by the leading evolution kernel singularities [72]. In a similar way, the non-singlet PDF combinations can be constrained by the infrared QCD evolution kernel [73]. An additional constraint may come from unitarity [74] and the universalities observed for the proton, photon, and diffractive structure functions [75]. In contrast, the NNPDF collaboration uses a very flexible form of PDF parameterisation, with 259 free parameters [7]. The gradient minimization methods are inapplicable for that large a number of parameters, and instead the NNPDF PDFs were fitted to the data using the neural network technique. Within the neural network approach, the PDF uncertainties are calculated from the probability distribution of the neural network replicas. Since no tensions between the different data sets used in the fit were observed (see Figure 7) the standard statistical methods are employed for the NNPDF PDFs. The uncertainties in the HERAPDF, ABKM, and JR PDFs are also calculated using the stan-

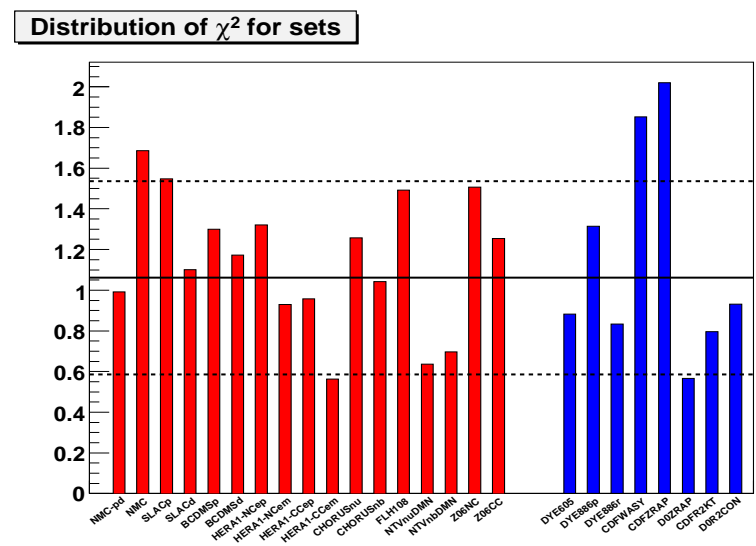

Figure 7: Distribution of the $\chi^{2} / N D P$ over the data sets used in the NNPDF2.0 fit. Figure is taken from Ref. [7]. 
dard statistical methods. For comparison, the CTEQ and MSTW collaborations apply larger tolerance factors to take into account discrepancies between the data sets used in the fit and theoretical uncertainties, such as parameterisation choices. Despite the different statistical treatments, the PDF errors provided by all groups are in qualitative agreement with each other.

Outside the kinematic region covered by the existing data, the NNPDF PDF uncertainties are larger than the other PDF uncertainties, due to the lack of prior theoretical/parameterisation constraints on the PDFs. On the other hand, the uncertainties for the JR PDFs are reduced, compared to other fits due to additional theoretical constraints. A study of the small- $x$ sea and gluon distribution flexibility allowed by the data was also performed with the PDFs parameterized by the Chebyshev polynomials [76,77]. The gluon distribution obtained in Ref. [76] is stable with respect to the polynomial powers used at $x \gtrsim 0.001$; at smaller $x$ the gluon distribution is unstable, due to the lack of the experimental constraints.

The PDFs given in Table 1 are similar, but do not completely overlap within $1 \sigma$ uncertainty bands. This discrepancy appears to be due to differences in the theoretical formalisms used in the global fits and in details of the data treatment; however, no definitive understanding has yet been reached. In addition, the treatment of the strong coupling constant $\alpha_{\mathrm{s}}$ is different for the PDFs listed in Table 1.

In Refs. $[2,5,6]$ the value of $\alpha_{\mathrm{s}}$ is fitted to the data simultaneously with the PDFs, while in the fits of Refs. [3, 4, 7] it is fixed at a value close to the world average [78]. For many cross sections, such as Higgs and top quark pair production, the rates are quite sensitive to the value of $\alpha_{\mathrm{s}}$ used (cf. Refs. [79-81]). This sensitivity, and the variations in the values of $\alpha_{s}$ used, results in additional uncertainties on the hard-scattering cross section predictions. For the NLO Higgs cross section benchmark, the spread of the predictions is as large as $20 \%$ (see Figure 8). Further consolidation of the predictions would make LHC predictions for the Higgs cross section more definitive.

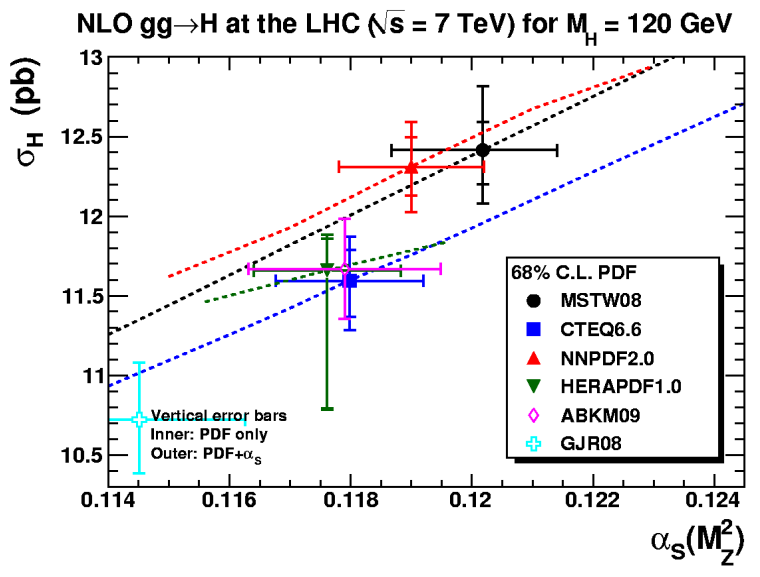

Figure 8: Comparison of the NLO Higgs rates at the LHC collision energy of $7 \mathrm{TeV}$ calculated with different PDFs. Figure is taken from Ref. [82]. The corresponding spread for predictions at the Tevatron can also be important for the interpretation of the Fermilab collider data [83].

The PDF4LHC working group is carrying out a benchmarking exercise [84] where each PDF group has been invited to provide NLO predictions for benchmark processes (such as the Higgs production cross sections for the Higgs masses of 120,180 and $240 \mathrm{GeV}$, as well as for $W, Z$ and $t \bar{t}$ production. The predictions are to be made for the default value of $\alpha_{s}$, as well as for a range of values from 0.116 to 0.118 . Comparisons are available at the website [85], along with a prescription for the calculation of the PDF uncertainties at the LHC. 
The last year has seen tremendous progress on both the theoretical and the experimental fronts. In particular, the release of the combined H1+ZEUS HERA I data has led to increased precision and thus better constraints on PDFs, especially the low- $x$ gluon distribution. The Tevatron Run II Wlepton asymmetry measurements have the potential to allow for improvements in the description of high- $x$ quark distributions, but conflict with some of the data sets currently in use in the global fits. A resolution of the conflict, perhaps with better understanding of some of the nuclear corrections for the fixed target data that conflict with the lepton asymmetry data, is needed.

The LHC is presenting its first results for standard model cross sections. Predictions for these cross sections are available using PDFs from a number of fitting groups. It is useful and important at this stage of LHC running to have benchmark comparisons of the predictions from the various PDF groups. Such an exercise has been carried out by the PDF4LHC working group. Further standardization would be extremely useful, in particular common estimate of the uncertainty in the value of $\alpha_{s}$.

At the next DIS workshop, we expect our first inputs from LHC data to PDF fitting. To significantly affect the existing PDF fits, the systematic errors must be reasonably small and well-known for the measurements to be included. The most likely cross sections to be used in such a way are Drell-Yan measurements, especially of $W$ and $Z$ boson production, with event yields of the order of a million events per experiment to be expected for $1 \mathrm{fb}^{-1}$, and with the possibility of accessing new kinematic regions in $x$ and $Q^{2}$ [86-88].

\section{References}

[1] Slides: http://indico.cern.ch/contributionDisplay.py?sessionId=4; contribId=364; confId=86184 http://indico.cern.ch/contributionDisplay.py?sessionId=4; contribId=365; confId =86184

[2] S. Alekhin, J. Blümlein, S. Klein and S. Moch, Phys. Rev. D 81 (2010) 014032.

[3] P. M. Nadolsky et al., Phys. Rev. D 78 (2008) 013004.

[4] F. D. Aaron et al. [H1 Collaboration and ZEUS Collaboration], JHEP 1001, 109 (2010).

[5] P. Jimenez-Delgado and E. Reya, Phys. Rev. D 79, 074023 (2009).

[6] A. D. Martin, W. J. Stirling, R. S. Thorne and G. Watt, Eur. Phys. J. C 63 (2009) 189.

[7] R. D. Ball, L. Del Debbio, S. Forte, A. Guffanti, J. I. Latorre, J. Rojo and M. Ubiali, Nucl. Phys. B 838 (2010) 136.

[8] S. Alekhin, arXiv:1008.3988 [hep-ph].

[9] J. Grebenyuk, these proceedings; http://indico.cern.ch/contributionDisplay.py?sessionId=4; contribId=170; confId=86184

[10] V. Radescu, these proceedings; http://indico.cern.ch/contributionDisplay.py?sessionId=4; contribId=318; confId=86184

[11] R. S. Thorne, arXiv:1006.5925 [hep-ph].

[12] M. A. G. Aivazis, J. C. Collins, F. I. Olness and W. K. Tung, Phys. Rev. D 50, 3102 (1994), M. A. G. Aivazis, F. I. Olness and W. K. Tung, Phys. Rev. D 50, 3085 (1994), 
[13] W. K. Tung, S. Kretzer and C. Schmidt, J. Phys. G 28, 983 (2002).

[14] K. Oliver, these proceedings; http://indico.cern.ch/contributionDisplay.py?sessionId=4; contribId=349; confId=86184

[15] S. Shushkevich, these proceedings; http://indico.cern.ch/contributionDisplay.py?sessionId=4; contribId=348; confId=86184

[16] V. Chekelian, these proceedings; http://indico.cern.ch/contributionDisplay.py?sessionId=4; contribId=350; confId=86184

[17] R. Ingbir, these proceedings; http://indico.cern.ch/contributionDisplay.py?sessionId=4; contribId=351; confId=86184

[18] ZEUS Collaboration. Measurement of Neutral Current Cross Sections at High Bjorken-x with the ZEUS Detector at HERA Eur. Phys. J. C49:523-544, 2009.

[19] Z. Zhang, these proceedings; http://indico.cern.ch/contributionDisplay.py?sessionId=24; contribId=352; confId=86184

[20] H1 Collaboration. A Determination of Electroweak Parameters at HERA, Phys. Lett. B 632 (2006) 35.

[21] The ALEPH Collaboration, the DELPHI Collaboration, the L3 Collaboration, the OPAL Collaboration, the SLD Collaboration, the LEP Electroweak Working Group, the SLD electroweak, heavy flavour groups. Precision Electroweak Measurements on the Z Resonance. Phys. Rept. 427:257 (2006), [arXiv:hep-ex/0509008v3].

[22] CDF Collaboration. Measurement of the Forward-Backward Charge Asymmetry of Electron-Positron Pairs in Proton anti-Proton Collisions at $s^{* *}(1 / 2)=1.96-T e V$. Phys. Rev. D71:052002 (2005), [arXiv:hep-ex/0411059v1].

[23] M. Klein, these proceedings; http://indico.cern.ch/contributionDisplay.py?sessionId=4; contribId=171; confId=86184

[24] H1 Collaboration. A Precision Measurement of the Inclusive ep Scattering Cross Section at HERA, Eur. Phys. J.C64:561-587,2009 [arXiv:0904.3513].

[25] A. Glazov, these proceedings; http://indico.cern.ch/contributionDisplay.py?sessionId=4; contribId=172; confId=86184

[26] H1 Collaboration. Measurement of the Inclusive ep Scattering Cross Section at Low $Q^{2}$ and $x$ at HERA, Eur.Phys.J.C63:625-678,2009 [arXiv:0904.0929].

[27] S. Habib, these proceedings; http://indico.cern.ch/contributionDisplay.py?sessionId=4; contribId=169; confId=86184

[28] R. S. Thorne, A. D. Martin, W. J. Stirling and G. Watt, arXiv:1006.2753 [hep-ph].

[29] H. L. Lai, M. Guzzi, J. Huston, Z. Li, P. M. Nadolsky, J. Pumplin and C. P. Yuan, arXiv:1007.2241 [hep-ph].

P. Nadolsky, these proceedings; http://indico.cern.ch/contributionDisplay.py?sessionId=4; contribId=175; confId=86184 M. Guzzi, these proceedings; http://indico.cern.ch/contributionDisplay.py?sessionId=4; contribId=176; confId=86184

[30] S. Alekhin, J. Blumlein and S. Moch, arXiv:1007.3657 [hep-ph].

[31] C. Adloff et al. [H1 Collaboration], Eur. Phys. J. C 21 (2001) 33; S. Chekanov et al. [ZEUS Collaboration], Eur. Phys. J. C 21, 443 (2001). 
[32] S. Moch, J. A. M. Vermaseren and A. Vogt, Nucl. Phys. B 688 (2004) 101; A. Vogt, S. Moch and J. A. M. Vermaseren, Nucl. Phys. B 691 (2004) 129.

[33] J. A. M. Vermaseren, A. Vogt and S. Moch, Nucl. Phys. B 724 (2005) 3; S. Moch and M. Rogal, Nucl. Phys. B 782 (2007) 51; S. Moch, M. Rogal and A. Vogt, Nucl. Phys. B 790 (2008) 317; S. Moch, J. A. M. Vermaseren and A. Vogt, Nucl. Phys. B 813 (2009) 220.

[34] E. Laenen, S. Riemersma, J. Smith and W. L. van Neerven, Nucl. Phys. B 392 (1993) 162.

[35] R.S. Thorne and W.K. Tung, in: Proceedings of the workshop: HERA and the LHC workshop series on the implications of HERA for LHC physics, eds. H. Jung et al., arXiv:0903.3861 [hep-ph].

[36] P. M. Nadolsky and W. K. Tung, Phys. Rev. D 79 (2009) 113014 [arXiv:0903.2667 [hep-ph]].

[37] W. K. Tung, H. L. Lai, A. Belyaev, J. Pumplin, D. Stump and C. P. Yuan, JHEP 0702 (2007) 053.

[38] A. M. Cooper-Sarkar, arXiv:1006.4471 [hep-ph].

[39] M. Buza, Y. Matiounine, J. Smith and W. L. van Neerven, Eur. Phys. J. C 1 (1998) 301; I. Bierenbaum, J. Blümlein and S. Klein, Phys. Lett. B 672 (2009) 401.

[40] S. Forte, E. Laenen, P. Nason and J. Rojo, Nucl. Phys. B 834, 116 (2010).

[41] A. Chuvakin, J. Smith and W. L. van Neerven, Phys. Rev. D 61, 096004 (2000).

[42] M. Gluck, E. Reya and M. Stratmann, Nucl. Phys. B 422, 37 (1994).

[43] I. Bierenbaum, J. Blümlein and S. Klein, Nucl. Phys. B820 (2009) 417.

[44] A. D. Martin, W. J. Stirling, R. S. Thorne and G. Watt, arXiv:1007.2624 [hep-ph].

[45] M. Dittmar, F. Pauss and D. Zurcher, Phys. Rev. D 56 (1997) 7284.

[46] G. Altarelli, R. K. Ellis and G. Martinelli, Nucl. Phys. B 157 (1979) 461; C. Anastasiou, L. J. Dixon, K. Melnikov and F. Petriello, Phys. Rev. D 69 (2004) 094008.

[47] T. Kluge, K. Rabbertz and M. Wobisch, arXiv:hep-ph/0609285.

[48] T. Carli et al., Eur. Phys. J. C 66 (2010) 503.

[49] H. Schellman, these proceedings; D. E. Acosta et al. [CDF Collaboration], Phys. Rev. D 71 (2005) 051104; V. M. Abazov et al. [D0 Collaboration], Phys. Rev. D 77 (2008) 011106; V. M. Abazov et al. [D0 Collaboration], Phys. Rev. Lett. 101 (2008) 211801.

[50] New Muon Collaboration, M. Arneodo etal, Phys. Lett. B686, 107 (1995), arXiv: hep-ph/9509406.

[51] BCDMS, A.C. Benvenuti etal, Phys. Lett. B223, 485 (1989).

[52] BCMDS, A.C. Benvenuti etal, Phys. Lett. B237, 592 (1990).

[53] S. Catani, G. Ferrera and M. Grazzini, JHEP 1005 (2010) 006.

[54] I. Schienbein, J. Y. Yu, C. Keppel, J. G. Morfin, F. Olness and J. F. Owens, Phys. Rev. D 77 (2008) 054013; K. Kovarik, arXiv:1006.4988 [hep-ph].

[55] K. J. Eskola, H. Paukkunen and C. A. Salgado, JHEP 0904 (2009) 065.

[56] M. Hirai, S. Kumano and T. H. Nagai, Phys. Rev. C 76 (2007) 065207.

[57] D. de Florian and R. Sassot, Phys. Rev. D 69 (2004) 074028. 
[58] S. A. Kulagin and R. Petti, Nucl. Phys. A 765 (2006) 126; S. A. Kulagin and R. Petti, Phys. Rev. D 76 (2007) 094023; S. A. Kulagin and R. Petti, arXiv:1004.3062 [hep-ph].

[59] J. Seely et al., Phys. Rev. Lett. 103 (2009) 202301;

D. Gaskel, these proceedings; http://indico.cern.ch/contributionDisplay.py?sessionId=4; contribId=320; confId=86184

[60] A. D. Martin, R. G. Roberts, W. J. Stirling and R. S. Thorne, Eur. Phys. J. C 23, 73 (2002).

[61] H. L. Lai et al., Phys. Rev. D 55, 1280 (1997).

[62] J. Pumplin, J. Huston, H. L. Lai, P. M. Nadolsky, W. K. Tung and C. P. Yuan, Phys. Rev. D 80, 014019 (2009) [arXiv:0904.2424 [hep-ph]].

[63] J. R. Gaunt and W. J. Stirling, JHEP 1003 (2010) 005 [arXiv:0910.4347 [hep-ph]].

[64] T. Gehrmann, arXiv:1007.2107 [hep-ph].

[65] M. Ciafaloni, D. Colferai, G. P. Salam and A. M. Stasto, JHEP 0708, 046 (2007) [arXiv:0707.1453 [hep-ph]].

[66] G. Altarelli, R. D. Ball and S. Forte, Nucl. Phys. B 799 (2008) 199.

[67] R. D. Ball et al. [The NNPDF Collaboration], Nucl. Phys. B 823, 195 (2009).

[68] F. Caola, S. Forte and J. Rojo, Phys. Lett. B 686 (2010) 127.

[69] L. V. Gribov, E. M. Levin and M. G. Ryskin, Phys. Rept. 100, 1 (1983).

[70] G. Diana, J. Rojo and R. D. Ball, arXiv:1006.4250 [hep-ph].

[71] A. Knutsson, these proceedings; http://indico.cern.ch/contributionDisplay.py?sessionId=4; contribId=191; confId=86184

[72] A. De Rujula, S. L. Glashow, H. D. Politzer, S. B. Treiman, F. Wilczek and A. Zee, Phys. Rev. D 10 (1974) 1649.

[73] B. Ermolaev, these proceedings; http://indico.cern.ch/contributionDisplay.py?sessionId=4; contribId=319; confId=86184

[74] N. Armesto, A. B. Kaidalov, C. A. Salgado and K. Tywoniuk, Phys. Rev. D 81 (2010) 074002; N. Armesto, A. B. Kaidalov, C. A. Salgado and K. Tywoniuk, arXiv:1003.2947 [hep-ph];

[75] T.Klimkovich, these proceedings; http://indico.cern.ch/contributionDisplay.py?sessionId=4; contribId=197; confId=86184

[76] V. Radescu, these proceedings; http://indico.cern.ch/contributionDisplay.py?sessionId=4; contribId=328; confId=86184

[77] J. Pumplin, arXiv:0909.5176 [hep-ph].

[78] S. Bethke, Eur. Phys. J. C 64 (2009) 689.

[79] A. D. Martin, W. J. Stirling, R. S. Thorne and G. Watt, Eur. Phys. J. C 64, 653 (2009) [arXiv:0905.3531 [hep-ph]].

[80] H. L. Lai, J. Huston, Z. Li, P. Nadolsky, J. Pumplin, D. Stump and C. P. Yuan, arXiv:1004.4624 [hep-ph].

[81] F. Demartin, S. Forte, E. Mariani, J. Rojo and A. Vicini, Phys. Rev. D 82 (2010) 014002. 
[82] G. Watt, PDF4LHC meeting, Mar 26 2010; http://indico.cern.ch/getFile.py/access?contribId=0;resId=0; materialId=slides; confId=87871

[83] J. Baglio and A. Djouadi, arXiv:1003.4266 [hep-ph].

[84] J. Huston, these proceedings; http://indico.cern.ch/contributionDisplay.py?sessionId=4; contribId=187; confId=8614

[85] https://wiki.terascale.de/index.php?title=PDF4LHC_WIKI

[86] J. M. Keaveney, these proceedings; http://indico.cern.ch/contributionDisplay.py?sessionId=4; contribId=181; confId=86184

[87] F. De Lorenzi, these proceedings; http://indico.cern.ch/contributionDisplay.py?sessionId=4; contribId=184; confId=86184

[88] B. Dahmes, these proceedings; http://indico.cern.ch/contributionDisplay.py?sessionId=4; contribId=183; confId=86184 